\title{
Mechanisms and challenges in translational stroke research
}

\author{
Eng H Lo, MingMing Ning
}

Massachusetts General Hospital, Harvard Medical School, Boston,

Massachusetts, USA

\section{Correspondence to} Dr Eng H Lo,

Neuroprotection Research Laboratory, Massachusetts General Hospital, Harvard Medical School, Boston, MA 02129, USA: Lo@helix.mgh.harvard.edu

Accepted 21 February 2016

Copyright @ 2016 American Federation for Medical Research

\section{CrossMark}

To cite: $\mathrm{LO} E \mathrm{H}_{\text {, }}$

Ning MM. J Investig Med

2016;64:827-829.

\begin{abstract}
Translating basic science advances into clinical meaning has been challenging for stroke research. This does not, however, mean that the investigation of basic mechanisms is irrelevant. Translation is difficult because the underlying mechanisms are complex and ill-defined. The original focus on neuroprotection has now evolved into a broader consideration of the role of non-neuronal cells in stroke pathophysiology. The neurovascular unit may provide a conceptual framework within which interactions between neural, glial, and vascular cells comprise a basis for function and dysfunction in the central nervous system. Importantly, these cell-cell signaling pathways are also biphasic in nature, that is, mechanisms that are deleterious in the acute phase may surprisingly be required for neurovascular remodeling and plasticity during stroke recovery. Furthermore, injury-into-repair gradients are significantly influenced by a host of modifying factors and comorbidities. Rigorous dissection of these complex and recursive mechanisms should be required before they can be rationally targeted for stroke.
\end{abstract}

\section{INTRODUCTION}

Stroke is a major health problem. It continues to be an important cause of mortality and morbidity. With an aging population, the incidence of stroke will surely continue to increase, with significant implications for both cerebrovascular disease and dementia. ${ }^{1}$ Therefore, the search for widely applicable and effective treatments for diverse patient populations remains an urgent unmet need.

In carefully selected patients with acute ischemic stroke, rapid thrombolysis with tissue plasminogen activator is a proven Food and Drug Administration (FDA) approved approach. ${ }^{2}$ Recently, a number of clinical trials now suggest that mechanical reperfusion with thrombectomy devices may also be efficacious in selected patient cohorts. ${ }^{3}$ This may represent a major advance in acute ischemic stroke care. Importantly, this may also represent an opportunity to further pursue combination therapies. Initial analyses suggest that there remains a 20-30\% mismatch between effective recanalization and clinical improvements, ${ }^{4}$ and there may also be up to 60-90 min worth of 'usable time' when the patient is already in the hospital before recanalization actually occurs. ${ }^{5}$ These scenarios may provide opportunities for further improving outcomes in a wider range of patients if one can design targeted therapies to further protect brain tissue while mechanical recanalization is taking place.

\section{NOT JUST NEUROPROTECTION}

The initial impetus for stroke therapeutics was triggered in part by the definition of excitotoxicity. $^{6}$ After cerebral ischemia, energetic perturbations lead to uncontrolled neuronal depolarization, disruption in glutamate transmitter homeostasis, and overactivation of $\mathrm{N}$-methyl-D-aspartate (NMDA), alpha-amino3-hydroxy-5-methyl-4-isoxazolepropionic acid (AMPA), and metabotropic glutamate receptors that ultimately lead to ionic imbalance and neuronal death. ${ }^{7}$ Furthermore, ischemic injury also perturbs mitochondrial function, leading to the generation of reactive oxygen and nitrogen species, all of which underlie oxidative stress and neuronal dysfunction. ${ }^{8}$ Finally, at least in some experimental model systems, cerebral ischemia may also lead to an upregulation of various programed cell death pathways, comprising apoptosis, autophagy, and necroptosis. ${ }^{9}$ In cell culture models of oxygen-glucose deprivation and animal models of focal cerebral ischemia, blockade of these various excitotoxic, oxidative stress, and cell death mechanisms appear to significantly reduce neuronal injury. However, none of these neuroprotective strategies have been proven effective in clinical trials. ${ }^{10}$

The lack of effective neuroprotection has been widely analyzed and debated. Perhaps the treatment time windows were overoptimistic in early clinical trials. Perhaps patient populations were not optimized in many of these prior 'take-all-comers' design. Perhaps the standard dichotomized $\chi^{2}$-modified Rankin score was not sensitive enough. Perhaps some of these neuroprotective compounds did not effectively penetrate the blood-brain barrier. Perhaps cell culture and animal models may not accurately capture all aspects of clinical stroke in humans. There will surely be many reasons involved.

Nevertheless, the lack of effective translation does not mean that the basic molecular and cellular mechanisms are incorrect or irrelevant. ${ }^{11} 12$ Perhaps the difficulty in translation means that the mechanisms of stroke pathophysiology are complex and remain ill-defined. Reversing an initial insult by restoring blood flow is an 'upstream' method with large effects 
in the right patients. However, the subtleties of 'downstream' neuroprotection may be more difficult to detect.

Over the past decade, the concept of the neurovascular unit has provided a framework for dissecting stroke mechanisms. ${ }^{6}{ }^{13}$ In its essence, the concept is simple. The central nervous system (CNS) works not just because neurons are firing action potentials. The whole system works because of intricate cell-cell signaling loops between multiple cells from neuronal, glial, and vascular compartments (figure 1). For example, proper neurotransmission requires a balance between neuronal release and glial reuptake mechanisms. The blood-brain barrier function requires crosstalk between the cerebral endothelium and pericytes and astrocytic end-feet. Renewal and homeostasis in white matter require crosstalk between endothelial cells and oligodendrocyte precursors. Even the revolutionary functional MRI method itself is predicated on crosstalk between neuronal activity and hemodynamic response. Similarly, dysfunction and disease are also mediated by crosstalk between all cell types in the brain. Hence, the pathophysiology of stroke (and perhaps all CNS disorders) cannot be effectively investigated without careful consideration of the entire neurovascular unit. ${ }^{14}$

\section{INJURY AND REPAIR}

The ischemic penumbra provides an important theoretical target in stroke. ${ }^{15}$ Within the ischemic core, blood flow deficits are severe and brain cells die rapidly. However, the penumbral areas surrounding this core are thought to be transiently sustained by collaterals, so the more moderate levels of ischemia allow some brains cells to temporarily survive. Of course, over time, the penumbra will collapse and brain cells will die if proper treatments are not provided.

The penumbra may represent a meta-stable state where recovery versus cell death are in dynamic and precarious balance. ${ }^{16}$ Importantly, the penumbra is both dying as well as actively trying to recover. ${ }^{17}$ The mammalian brain

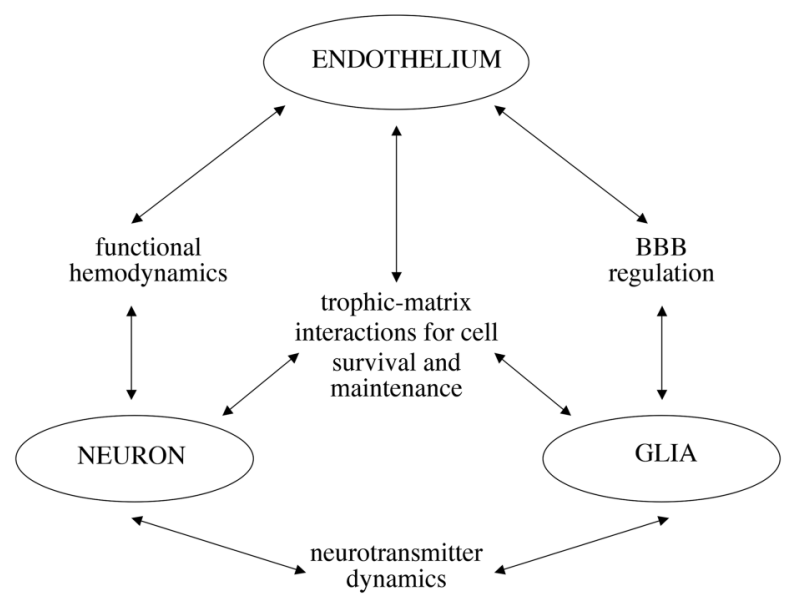

Figure 1 The neurovascular unit provides a conceptual framework that emphasizes the importance of cell-cell interactions for central nervous system function. Interactions between neurons and glia sustain neurotransmission. Interactions between the endothelium and glia sustain the blood-brain barrier function. Interactions between neurons and the endothelium sustain metabolic-hemodynamic coupling. BBB, blood-brain-barrier. possesses remarkable endogenous mechanisms for compensation, plasticity, and remodeling. Stroke mechanisms may be interpreted under a general rubric of inflammation and wound healing. Many mediators in the pathophysiology of stroke and CNS disease come from the larger families of damage-associated molecular pattern (DAMP) and pathogen-associated molecular pattern (PAMP) signals. ${ }^{18}$ Hence, the numerous pathways that are triggered after stroke may possess biphasic properties. For example, overactivation of NMDA receptors will cause excitotoxicity and neuronal death in the acute phase, but without proper NMDA signaling, recovery cannot take place when patients move into rehabilitation units. ${ }^{17}$ Uncontrolled oxidative stress is damaging but homeostatic levels of free radicals are required for angiogenesis and endothelial progenitor cell function. ${ }^{19}$ Programed cell death pathways may also be essential for maintaining functional cell subsets, and deletion of autophagy genes may induce neurodegeneration. ${ }^{20}$ Microglia are not always damaging; M1-like subsets are deleterious, whereas M2-like subsets may promote tissue repair. $^{21}$

Hence, targeting the penumbra in stroke and, perhaps more broadly, treatment of any CNS disorder may require careful attention to the signals involved as the brain transitions from initial injury into subsequent endogenous modes of neurovascular remodeling and recovery. ${ }^{22}$

\section{COFACTORS AND COMORBIDITIES}

Stroke pathophysiology is complex because it involves more than just metabolically dying neurons. Crosstalk between neuronal, glial, and vascular compartments plays a critical role. ${ }^{6}$ Recently, however, it is recognized that the brain is not an 'isolated organ', so an 'extended' neurovascular unit has been proposed. ${ }^{11}$ Experimental and clinical data strongly suggest interactions between the CNS and peripheral responses from immune, hormonal, and cardiac systems. The spleen, bone marrow, and circulating immune cells are all activated after stroke. ${ }^{23}$ Changes in the brain are correlated with changes in the heart. ${ }^{24}$

Crosstalk between the CNS and peripheral response may provide the potential signals and substrates that permit the influence of many cofactors and comorbidities (figure 2). Genome-wide-association-studies (GWAS) suggest that many stroke genes may represent cardiovascular pathways. ${ }^{25}$ Hypertension and diabetes have long been known to be known to be significant factors in stroke. ${ }^{26}$ In fact, many of these modifying factors alter stroke risk, as well as significantly contribute to the acute process of brain injury and the delayed process of brain repair. For example, it has been suggested that diabetic vascular systems may suffer

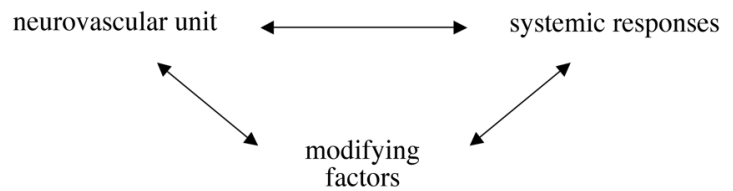

Figure 2 The neurovascular unit also interacts with systemic responses after stroke comprising cardiac, hormonal, and immune mediators. The influence of typical stroke risk factors (eg, hypertension, diabetes) are often manifested as modifying mechanisms that influence central-systemic crosstalk signals. 
from impaired angiogenic programs that might influence stroke recovery. ${ }^{27}$

From the experimental side, model systems will have to be adapted to incorporate these important modifying factors. From the clinical side, stroke trials may have to more carefully identify and separate patient cohorts depending on how the mechanisms being targeted are affected by various risk factors. Ultimately, a systems biology approach may be helpful. For example, mapping the transcriptome of reactive astrocytes should offer insight into how deleterious versus pro-recovery gliosis may be regulated for therapeutic benefit. ${ }^{28}$ Recent attempts to map the brain vasculome should also provide a rich database for dissecting new mechanisms and stroke targets in susceptible patient populations. ${ }^{29} 30$

\section{LOOKING AHEAD}

A challenging problem is not necessarily intractable. The recent success of endovascular trials suggests that we can indeed design clinical trials that select patients for treatment in a timely manner. Once patients are in this system, time windows do exist for combination therapies to be contemplated. The completion of the Field Administration of Stroke Therapy-Magnesium (FAST-MAG) trial further suggests that prehospital treatments may even be considered under the right circumstances. Advances in neuroimaging suggest that rapid methods to separate responders from non-responders may perhaps be feasible in the near future. Ultimately, rigorous attempts to define and link mechanisms across all cell, animal, and human model systems will be essential. ${ }^{31-33}$ Stroke remains a difficult disease with heterogeneous patient populations. However, with a rigorous dissection of causal mechanisms, translation may still be possible.

Contributors $\mathrm{EHL}$ wrote this article in consultation with MN.

Competing interests None declared.

Provenance and peer review Commissioned; externally peer reviewed.

\section{REFERENCES}

1 Gorelick PB, Scuteri A, Black SE, et al. American Heart Association Stroke Council, Council on Epidemiology and Prevention, Council on Cardiovascular Nursing, et al. Vascular contributions to cognitive impairment and dementia: a statement for healthcare professionals from the American Heart Association/American Stroke Association. Stroke 2011;42:2672-713.

2 Campbell BC, Meretoja A, Donnan GA, et al. Twenty-year history of the evolution of stroke thrombolysis with intravenous alteplase to reduce long-term disability. Stroke 2015;46:2341-6.

3 Yarbrough $C K$, Ong $C J$, Beyer $A B$, et al. Endovascular thrombectomy for anterior circulation stroke: systematic review and meta-analysis. Stroke 2015;46:3177-83. Erratum in: Stroke 2015;46:e249.

4 Grotta JC, Hacke W. Stroke neurologist's perspective on the new endovascular trials. Stroke 2015;46:1447-52.

5 Menon BK, Campbell BC, Levi C, et al. Role of imaging in current acute ischemic stroke workflow for endovascular therapy. Stroke 2015;46:1453-61.
6 Lo EH, Dalkara T, Moskowitz MA. Mechanisms, challenges and opportunities in stroke. Nat Rev Neurosci 2003;4:399-415.

7 Lipton P. Ischemic cell death in brain neurons. Physiol Rev 1999;79:1431-568.

8 Chan PH. Reactive oxygen radicals in signaling and damage in the ischemic brain. J Cereb Blood Flow Metab 2001;21:2-14.

9 Yuan J, Yankner BA. Apoptosis in the nervous system. Nature 2000;407:802-9.

10 O'Collins VE, Macleod MR, Donnan GA, et al. 1,026 Experimental treatments in acute stroke. Ann Neurol 2006;59:467-77.

11 Lo EH. 2013 Thomas Willis Award Lecture: causation and collaboration for stroke research. Stroke 2014;45:305-8.

12 Fisher M. The spectrum of translational stroke research. Neurol Res 2013;35:443-7.

13 Lo EH, Broderick JP, Moskowitz MA. tPA and proteolysis in the neurovascular unit. Stroke 2004;35:354-6.

14 Guo S, Lo EH. Dysfunctional cell-cell signaling in the neurovascular unit as a paradigm for central nervous system disease. Stroke 2009;40(3 Suppl): S4-7.

15 Heiss WD, Graf R. The ischemic penumbra. Curr Opin Neurol 1994;7:11-19.

16 von Bornstädt D, Houben T, Seidel JL, et al. Supply-demand mismatch transients in susceptible peri-infarct hot zones explain the origins of spreading injury depolarizations. Neuron 2015;85:1117-31.

17 Lo EH. A new penumbra: transitioning from injury into repair after stroke. Nat Med 2008;14:497-500

18 Xing C, Hayakawa K, Lok J, et al. Injury and repair in the neurovascular unit. Neurol Res 2012;34:325-30.

19 Ushio-Fukai M. Redox signaling in angiogenesis: role of NADPH oxidase. Cardiovasc Res 2006;71:226-35.

20 Komatsu M, Waguri S, Chiba T, et al. Loss of autophagy in the central nervous system causes neurodegeneration in mice. Nature 2006;441:880-4.

21 Hu X, Leak RK, Shi Y, et al. Microglial and macrophage polarization-new prospects for brain repair. Nat Rev Neurol 2015;11:56-64.

22 Lo EH. Degeneration and repair in central nervous system disease. Nat Med 2010;16:1205-9.

23 ladecola C, Anrather J. The immunology of stroke: from mechanisms to translation. Nat Med 2011;17:796-808.

24 Ishikawa H, Tajiri N, Vasconcellos J, et al. Ischemic stroke brain sends indirect cell death signals to the heart. Stroke 2013;44:3175-82.

25 Markus HS, Bevan S. Mechanisms and treatment of ischaemic strokeinsights from genetic associations. Nat Rev Neurol 2014;10:723-30.

26 Mozaffarian D, Benjamin EJ, Go AS, et al. American Heart Association Statistics Committee and Stroke Statistics Subcommittee. Executive summary: heart disease and stroke statistics-2016 update: a report from the American Heart Association. Circulation 2016;133:447-54.

27 Ergul A, Valenzuela JP, Fouda AY, et al. Cellular connections, microenvironment and brain angiogenesis in diabetes: Lost communication signals in the post-stroke period. Brain Res 2015;1623:81-96.

28 Zamanian JL, Xu L, Foo LC, et al. Genomic analysis of reactive astrogliosis. J Neurosci 2012;32:6391-410.

29 Guo S, Lok J, Zhao S, et al. Effects of controlled cortical impact on the mouse brain vasculome. J Neurotrauma 2016.

30 Guo S, Zhou Y, Xing C, et al. The vasculome of the mouse brain. PLOS ONE 2012;7:e52665.

31 Lopez MF, Krastins B, Sarracino DA, et al. Proteomic signatures of serum albumin-bound proteins from stroke patients with and without endovascular closure of PFO are significantly different and suggest a novel mechanism for cholesterol efflux. Clin Proteomics 2015;12:2.

32 Ning M, Lo EH, Ning PC, et al. The brain's heart-therapeutic opportunities for patent foramen ovale (PFO) and neurovascular disease. Pharmacol Ther 2013;139:111-23.

33 Ning M, Sarracino DA, Kho AT, et al. Proteomic temporal profile of human brain endothelium after oxidative stress. Stroke 2011;42:37-43. 